\title{
Composés organiques volatils : des mécanismes moléculaires intriqués au centre de la complexité de la chimie troposphérique
}

Jean-François Doussin

Laboratoire interuniversitaire des systèmes atmosphériques, Institut PierreSimon Laplace, CNRS / Université Paris Est Créteil / Université de Paris, Créteil

Jean-francois.doussin@lisa.ipsl.fr

\section{Résumé}

La pollution de l'air demeure l'un des principaux fléaux des temps modernes. Outre la pollution atmosphérique dite "primaire " se développe aussi une pollution atmosphérique plus pernicieuse, appellée « secondaire ", produite dans l'environnement atmosphérique. Elle est le fruit d'une chimie atmosphérique multiphasique, impliquant des composés organiques et radicalaires, rendue complexe à la fois par le grand nombre de composants de l'air et par la multiplicité de leurs voies d'évolution chimique. En mettant en perspective cette complexité, cet article se propose de donner quelques clefs pour l'appréhender et de présenter les stratégies de la recherche qui permettront de réduire cette pollution secondaire.
L ongtemps, on a considéré notre atmosphère comme infinie. À peine avait-on pris conscience de sa finitude, depuis les travaux de Florin Périer au XVII ${ }^{\mathrm{e}}$ siècle à ceux de Laplace au début du XIX $^{\mathrm{e}}$ siècle, qu'il aurait fallu en considérer les limites. Bien au contraire, elle est devenue le réceptacle des déchets de l'activité humaine, et notamment ceux des combustibles fossiles, depuis la révolution industrielle. Pourtant, de l'accident de la vallée de la Meuse en 1930 aux brouillards sulfureux de Londres (Bates et al., 2002), nous aurions pu en saisir la vulnérabilité. Ce n'est que dans les années 19701980 avec les découvertes de l'impact global des chlorofluorocarbures (CFC), de l'accumulation du $\mathrm{CO}_{2}$ et de l'intensification des événements de pollution photochimique dans les mégapoles qu'il est devenu évident que la dilution des polluants atmosphériques n'équivalait pas à leur élimination. Aujourd'hui, pour chacun d'entre nous, il est admis que ce que nous émettons se retrouve dans l'air que nous respirons, même dans les zones les plus reculées et dans les hautes couches de l'atmosphère.

\section{Qu'entend-on par pollution atmosphérique?}

Même si intuitivement la pollution atmosphérique semble un concept bien établi, sa définition varie de telle manière qu'il est finalement peu utile aux scientifiques. Pour certains, il s'agit de la présence d'impuretés dans l'air ou l'élévation « anormale » de la proportion de certains constituants de l'atmosphère. Cette définition fait référence à une composition «normale » de l'air qui aurait, peut-être, préexisté aux émissions liées aux activités humaines. Cet état imaginaire de l'atmosphère n'est que de peu d'utilité aux chimistes de l'atmosphère tant il est vrai qu'en l'absence d'émissions anthropiques, il perdure une chimie atmosphérique très active et suivant sensiblement les mêmes voies de celle «perturbée » d'aujourd'hui.

Mêlant à la fois origine anthropique et impact nocifs, la loi sur l'air et l'utilisation rationnelle de l'énergie du 30 décembre 1996 propose la définition suivante: "Constitue une pollution atmosphérique [...] l'introduction par l'homme, directement ou indirectement, dans l'atmosphère et les espaces clos, de substances ayant des conséquences préjudiciables de nature à mettre en danger la santé humaine, à nuire aux ressources biologiques et aux écosystèmes, à influer sur les changements climatiques, à détériorer les biens matériels, à provoquer des nuisances olfactives excessives. »

Gardant en ligne de mire ces impacts pour contribuer à les réduire, les chimistes de l'atmosphère, considèrent, pour leur part, l'atmosphère comme un réacteur où se mélangent et interagissent sans distinction espèces d'origine anthropique et d'origine naturelle. 


\section{Abstract}

Volatile organic compounds: Intricate molecular mechanisms at the center of the complexity of tropospheric chemistry

Air pollution remains one of the main plagues of modern times. In addition to so-called "primary" air pollution, a more pernicious air pollution is also developing, termed "secondary", i.e. produced in the atmospheric environment. It is the result of an atmospheric multiphase chemistry involving organic and radical compounds, made complex both by the large number of components in the air and the multiplicity of their chemical pathways. Putting this complexity into perspective, this article provides some keys to understand it and to present research strategies that will reduce this secondary pollution.
Cependant, à côté de la pollution dite « primaire » (car directement émise dans l'atmosphère) se développe une pollution plus pernicieuse, dite secondaire, car produite dans l'atmosphère elle-même. C'est la chimie atmosphérique qui en est responsable.

Pour s'en convaincre, il suffit de considérer combien les pics estivaux d'ozone constituent un problème majeur, alors même qu'aucun émetteur significatif d'ozone n'est recensé.

Extrêmement complexe du fait, entre autres, de la diversité et du nombre d'espèces chimiques impliquées, non linéaire, souvent hors équilibre et dépendant des conditions environnementales, la chimie troposphérique est essentielle pour comprendre et anticiper l'impact des activités humains sur la qualité de l'air (aux échelles régionales et locales) et sur le climat (à l'échelle globale).

D'un point de vue macroscopique, le rayonnement solaire en constitue la force motrice puisque la plupart des réactions qui assurent l'initiation ou la propagation des cycles catalytiques qui $\mathrm{y}$ règnent sont photolytiques. Si le soleil fournit l'énergie qui permet de rompre les premières liaisons chimiques et donc d'initier ces transformations chimiques, c'est la combinaison des fragments de molécules avec le dioxygène de l'air qui en oriente le résultat : les polluants subissent une oxydation progressive.

\section{L'atmosphère : un réacteur chimique complexe}

Parmi les composés organiques, certains sont suffisamment volatils pour être présents dans l'atmosphère sous forme gazeuse. Imbrûlés de combustion, solvants, rejets industriels, mais aussi molécules émis par la végétation, ces composés organiques volatils (COV) sont " attaqués » par des radicaux libres dont les principaux sont $\mathrm{OH}^{\bullet}$, et $\mathrm{NO}_{3}^{\bullet}$ et l'ozone $\left(\mathrm{O}_{3}\right)$, pour être eux-mêmes transformés en radicaux se combinant à l'oxygène de l'air et formant ainsi des polluants secondaires plus oxydés et souvent plus toxiques.

Le jour, les radicaux organiques convertissent le monoxyde d'azote (NO) en dioxyde d'azote $\left(\mathrm{NO}_{2}\right)$, dont la photolyse régénère le premier, et une molécule d'ozone. Or, les composés secondaires ainsi produits peuvent, à leur tour, alimenter ces cycles photocatalytiques. La combinaison des oxydes d'azote (principalement émis par les processus de combustion, dont le transport routier) et du rayonnement solaire permet ainsi l'oxydation progressive des $\mathrm{COV}$, chaque degré d'oxydation ainsi franchi conduisant à la formation d'une molécule d'ozone, qui s'accumule tant que des réactions secondaires ou leur dépôt sur les surfaces ne viennent en limiter la concentration.

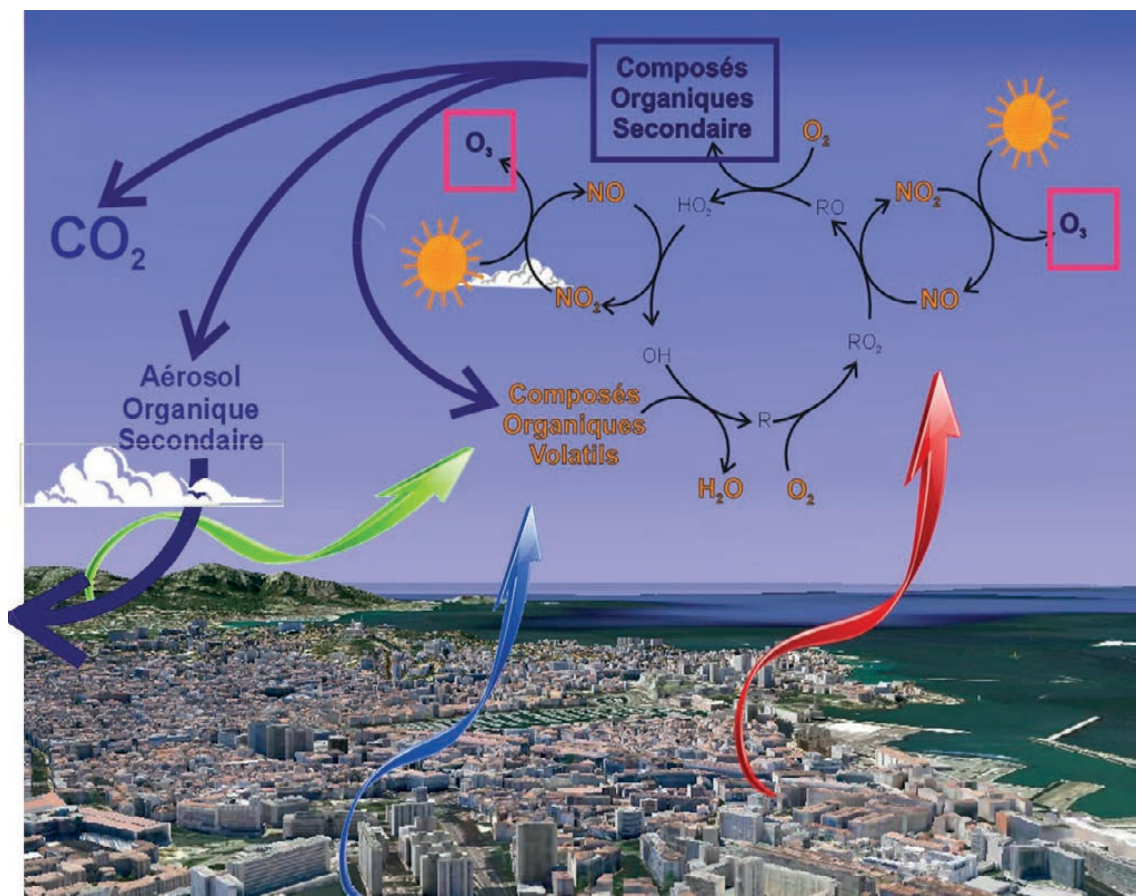

Figure 1. Mécanisme général de l'oxydation des composés organiques volatils initiée par le radical $\mathrm{OH}$ et conduisant à l'accumulation d'ozone et à l'oxydation de la matière organique. 
Ce mécanisme général (figure 1) explique l'accumulation de l'ozone, ce gaz hautement irritant responsable des pics de pollution de nos fins d'après-midi d'été.

$\mathrm{Au}$ cours de leurs transformations atmosphériques, les polluants organiques voient généralement leur structure débitée en une succession de molécules plus petites dont l'étape ultime est la formation de $\mathrm{CO}_{2}$. On a longtemps cru que cette dernière étape était la conséquence majeure de ces processus d'oxydation progressive des polluants organiques, au point que le radical $\mathrm{OH}^{\bullet}$ était parfois qualifié de " nettoyeur de l'atmosphère " et que le concept confortable d'auto-épuration de l'atmosphère était souvent avancé.

Cette supposition n'est que partiellement vraie et notre compréhension de ces mécanismes, en s'affinant ces dernières décennies, a révélé un tableau plus complexe encore. Il a en effet été mis en évidence que les chaînes radicalaires qui apparaissent après l'attaque d'un COV ne conduisent pas nécessairement à la fragmentation de celui-ci et qu'il existait des réactions d'isomérisation permettant la conservation du squelette carboné. De cette deuxième voie, qui s'est avérée majeure pour des molécules présentant une chaîne de cinq carbones et plus, découle la formation de produits secondaires dont la faible volatilité ne permet plus qu'ils restent uniquement en phase gazeuse. Ces produits condensent et viennent augmenter la fraction fine de l'aérosol atmosphérique. On parle alors d'aérosol organique secondaire. Ces aérosols contribuent notablement à la pollution aux «particules fines » et sont encore très mal représentés dans les modèles de qualité de l'air.

Les transformations troposphériques peuvent ainsi conduire à la production d'ozone, mais aussi d'espèces gazeuses secondaires très diverses tels des aldéhydes, des cétones, des composés nitrés, des acides ou encore à d'autres aérosols organiques secondaires. Ces espèces présentent des toxicités souvent aiguës qui participent à la dégradation de la qualité de l'air, à l'échelle locale ou régionale. Elles ont également des impacts radiatifs plus ou moins directs qui, à plus grande échelle, participent à la modification du climat. Cette double nocivité potentielle explique l'intérêt que suscite depuis des décennies l'étude du réacteur chimique atmosphérique. Or, plusieurs facteurs participent à sa complexité et

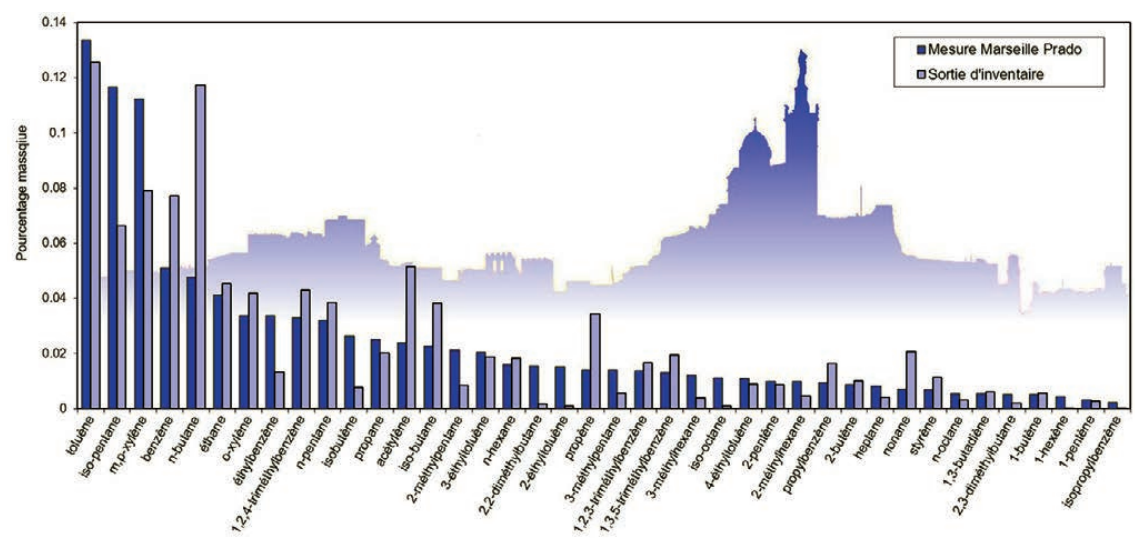

Figure 2. Inventaire d'émissions anthropiques : pourcentage massique pour 40 composés organiques volatils tel que mesuré et calculé. Sortie d'inventaire comparée aux mesures pendant la campagne Escompte conduite à Marseille à l'été 2001 (adapté de Coll et al., 2010). font de sa modélisation numérique un défi qui nécessite la combinaison de savoirs pluridisciplinaires.

\section{Diversité des émissions}

Le premier de ces facteurs est la multiplicité des émissions. En effet, les activités humaines, mais aussi les métabolismes végétaux, voire l'environnement marin ou le volcanisme, sont émetteurs de plusieurs milliers de composés organiques volatils différents. Dans la plupart des zones du globe, au-dessus de quelques centaines de ppt (part per trillion, ou partie par millier de milliards), on peut compter plusieurs dizaines à plusieurs centaines d'espèces différentes qui constituent aussi bien en zone anthropisée ou non des mélanges réactionnels extrêmement complexes. Ainsi, la figure 2 ne présente pas moins de 40 COV mesurés en centre-ville à Marseille pendant la campagne Escompte 2001, lors de laquelle ont été réalisés des cadastres d'émissions indispensables à l'alimentation des modèles tridimensionnels.

Ces inventaires sont en outre à considérer de manière dynamique. Les facteurs d'émissions des différentes

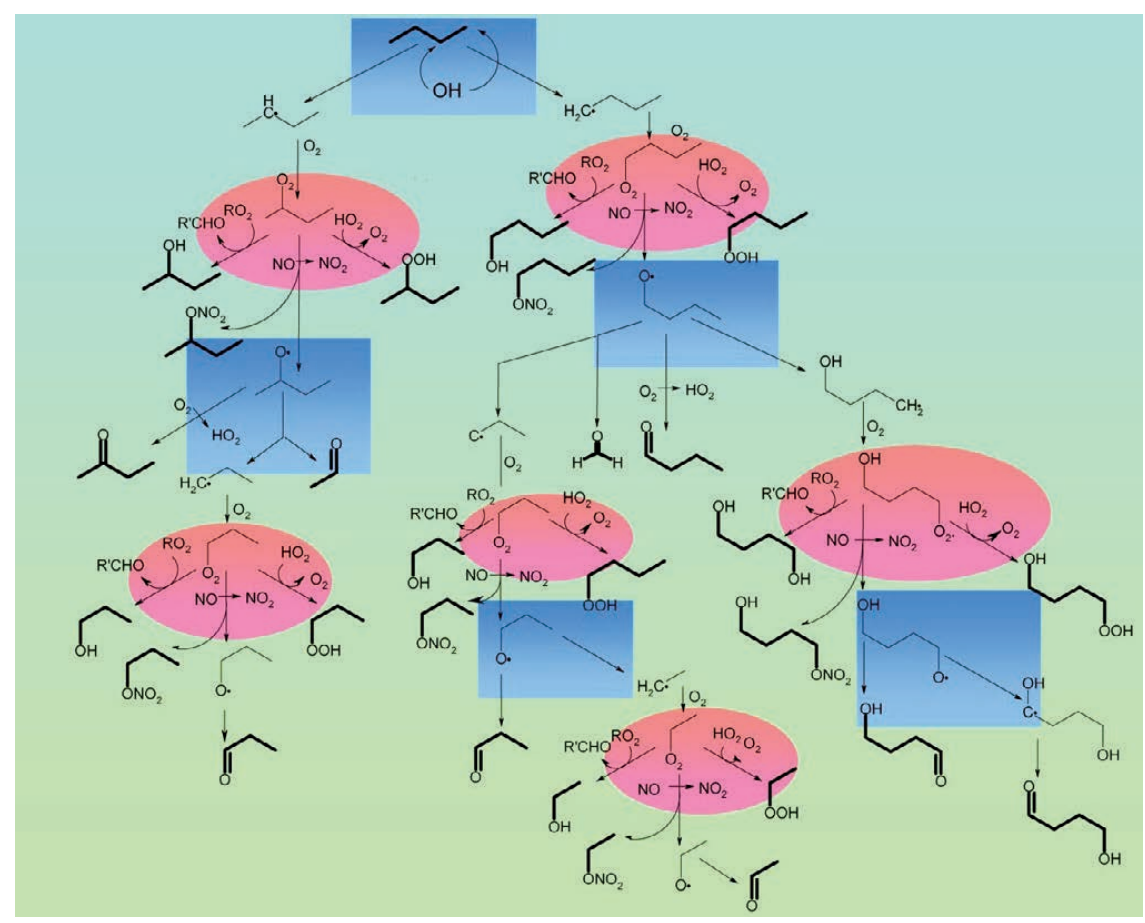

Figure 3. Schéma simplifié de l'oxydation atmosphérique du n-butane provoquée par le radical OH. Les composés indiqués en gras sont des molécules stables et constituent donc les polluants secondaires issus directement du $n$-butane.Les autres sont des intermédiaires radicalaires dont la durée de vie est extrêmement courte. Les branchements signalés en bleu sont essentiellement dépendants de la structure chimique de l'espèce considérée, alors que ceux en rouge sont au contraire essentiellement dépendants des conditions environnementales (concentration en oxydes d'azote). 
espèces varient dans le temps avec la variation des activités et des pratiques qui leur donnent naissance. Bien entendu, largement dépendants de l'occupation des sols, de l'aménagement et des zones d'activités, ils varient également dans l'espace.

\section{Multiplicité des transformations}

À cette complexité résultant des émissions il faut ajouter celle inhérente aux mécanismes chimiques en jeu et, en particulier, à la très grande diversité des voies d'évolution d'un seul composé organique. À titre d'exemple et afin d'illustrer cette diversité sont représentées, sur la figure 3 , les voies d'évolution du $n$-butane après son attaque par le radical $\mathrm{OH}^{\bullet}$. On a veillé à ne représenter que la première étape d'oxydation de ce COV de structure assez simple et, pourtant, on observe déjà la production de près de 22 composés secondaires distincts. Comme pour la quasi-totalité des COV, le schéma d'oxydation est hautement ramifié et présente un grand nombre d'embranchements dont l'importance relative est déterminante pour la distribution des produits secondaires. En l'occurrence, on peut distinguer deux types de branchement : i) ceux qui ne dépendent que de la structure moléculaire de l'espèce considérée (en bleu sur la figure 3) et ii) ceux qui dépendent fortement des conditions environnementales (en rouge).

Parmi les étapes qui ne dépendent que de la structure moléculaire de l'espèce impliquée, il faut distinguer l'attaque initiale des processus de propagation.

L'attaque initiale sur le COV considéré ne dépend que de la structure de celui-ci et de la nature du radical agresseur. Les radicaux $\mathrm{OH}^{\bullet}, \mathrm{NO}_{3}^{\bullet}$ et $\mathrm{Cl}^{\bullet}$ peuvent ainsi aussi bien s'additionner sur les doubles liaisons qu'arracher un atome d'hydrogène sur la molécule. Bien qu'ils présentent des sélectivités variables, leur réactivité est, d'une manière générale, fortement gouvernée par la structure du COV, puisque leurs sites d'attaque sur la molécule se situent préférentiellement sur les sites riches en électrons. L'ozone, au contraire, ne peut que s'additionner sur les doubles liaisons carbone-carbone ; il n'est donc réactif que vis-à-vis des espèces insaturées et la diversité de ses sites d'attaque est pratiquement nulle, sauf quand la molécule présente plusieurs doubles liaisons.
Cette diversité physico-chimique se combine avec une variabilité spatio-temporelle : $\mathrm{OH}^{\bullet}$ et $\mathrm{Cl}^{\bullet}$ nécessitant des sources photochimiques, ils constituent des agresseurs efficaces essentiellement le jour, alors que $\mathrm{NO}_{3}^{\bullet}$ n'est, lui, significatif que la nuit; l'ozone, en revanche, peut être présent de jour comme de nuit.

Cette étape d'attaque initiale est cinétiquement limitante, c'est-à-dire que c'est elle qui impose sa vitesse à l'ensemble du processus et détermine donc la durée de vie des composés organiques dans l'environnement atmosphérique. En effet, les réactions radicalaires qui en découlent sont si rapides que leur succession peut, à l'échelle des transformations atmosphériques des composés stables, être considérée comme instantanée.

Parmi les réactions subséquentes à l'attaque initiale, il en est qui sont également uniquement dépendantes de la structure moléculaire des intermédiaires impliqués. Il s'agit de celles pilotant le devenir des radicaux alcoxyles $\mathrm{RO} \bullet$ (également indiquées en bleu sur la figure 3). Cette étape présente plusieurs chemins réactionnels en compétition. D'une façon générale, on a répertorié trois voies possibles:

- arrachement d'un hydrogène voisin $\mathrm{du}$ groupement $\mathrm{C}=\mathrm{O}$ conduisant à la production d'un radical $\mathrm{HO}_{2}$ et d'un composé stable carbonylé (généralement un aldéhyde ou une cétone) ;

- décomposition du radical alcoxyle conduisant, d'une part, à la formation d'un radical alkyle et, d'autre part, à celle d'un aldéhyde ;

- dans le cas de radicaux alcoxyles longs (plus de quatre atomes de carbone), réarrangement interne conduisant à un radical alkyle hydroxylé.

Les importances relatives de ces différentes voies influent donc directement sur la nature des espèces stables produites. Elles intéressent ainsi particulièrement les problèmes de qualité de l'air. Par ailleurs, la réactivité des composés organiques secondaires susceptibles d'être ainsi produits peut varier de plusieurs ordres de grandeur. Les rapports de branchement des différentes voies influent donc sur l'activation des processus photochimiques.

Il faut cependant porter une attention particulière au troisième processus (branchements de droite sur la figure 3) qui, lorsqu'il est structurellement possible, est considéré aujourd'hui comme le processus majoritaire. En effet, alors que les deux autres voies conduisent à la fragmentation de la chaîne carbonée du COV initial, cette dernière voie en permet la conservation et plus encore la fonctionnalisation. Il est ainsi aisé, sur le schéma d'oxydation du $n$-butane, de constater que les seuls composés secondaires qui portent plusieurs fonctions chimiques $\left(\mathrm{OH}, \mathrm{C}=\mathrm{O}\right.$ ou $\left.\mathrm{ONO}_{2}\right)$ sont passés à un certain moment de leurs schémas d'oxydation par une étape d'isomérisation d'un radical alcoxyle. Cette étape est donc la clef de la fonctionnalisation de la matière organique atmosphérique qui n'a été mis en évidence que très récemment et qui permet la formation de produits secondaires présentant des volatilités de plus en plus faibles et donc conduisant à la production d'aérosol organique secondaire.

Enfin, il serait illusoire de penser que la distribution des produits d'oxydation de la matière organique secondaire atmosphérique serait entièrement prédéterminée par la structure chimique des composés primaires et par la présence ou non des radicaux agresseurs. En effet, les conditions environnementales influent fortement sur le déroulement des chaînes radicalaires. Dans le schéma d'oxydation du $n$-butane pris ici en exemple, cet effet environnemental est illustré par une étape essentielle : la réduction des radicaux peroxyles $\mathrm{RO}^{\bullet}$ (indiquée en rouge sur la figure 2 ). Cette étape est fondamentale, car c'est elle qui assure l'oxydation de $\mathrm{NO}$ en $\mathrm{NO}_{2}$ et permet l'accumulation d'ozone (voir figure 1). Cependant, elle joue également un grand rôle dans la distribution des composés secondaires et sa variabilité. En présence d'oxyde d'azote en quantité suffisante, c'est-à-dire près des sources en zone urbaine ou péri-urbaine, la réaction des $\mathrm{RO}_{2}^{\bullet}$ avec NO est majoritaire. Elle conduit à la formation de nitrates organiques (minoritaires) et, par l'intermédiaire de la chimie des $\mathrm{RO}^{\bullet}$ à des composés carbonylés (portant la fonction $-\mathrm{C}=\mathrm{O}$ ). Cependant, loin des sources, quand les concentrations de monoxyde d'azote sont de l'ordre de quelques dizaines de ppt - ce qui est le cas dans la majeure partie de la troposphère - les radicaux peroxyles ne sont plus significativement réduits par NO, mais au contraire par réaction sur eux-mêmes ou sur un radical $\mathrm{HO}_{2} \bullet$. Il s'ensuit la production d'alcools ou d'hydroperoxydes organiques $(\mathrm{ROOH})$, composés très solubles qui peuvent se dissoudre dans les gouttes nuageuses. Ces voies réactionnelles conduisent en outre à la réduction du nombre d'espèces radicalaires ; il s'agit donc de réaction de terminaison des cycles photochimiques dont le résultat est une consommation de l'ozone. 
Comme on peut le constater, la chimie d'une seule espèce assez simple par sa structure conduit déjà à une diversité considérable de produits de première génération dont les taux de formation ne sont pas prédéterminés, mais dépendent, en plus, des conditions environnementales. Il faut cependant bien garder à l'esprit que chacun de ces produits de première génération va également être soumis aux conditions oxydantes de l'atmosphère, être oxydé et finalement conduire à la production d'espèces de troisième, quatrième, cinquième... générations.

\section{La puissance informatique pour traiter la diversité chimique}

Alimenté par les expériences de laboratoire et les observations de terrain, le développement récent de schémas chimiques dits explicites permet aujourd'hui de prendre la mesure de l'explosion de cette diversité chimique. Comme le présente la figure 4, chaque composé injecté dans l'atmosphère va donner naissance à une myriade de composés secondaires dont le nombre suit une loi de puissance du nombre d'atomes de carbone présents dans la molécule.

La complexité combinée des émissions et des processus de transformation conduit à des relations et à des interdépendances dont la prise en compte implique obligatoirement l'établissement de modèles physico-chimiques très élaborés (figure 5). On y constate que la prise en compte de sept molécules seulement conduit déjà à des milliers de processus totalement interconnectés.

\section{Dénouer l'écheveau par la synergie des approches scientifiques}

Pour dénouer l'écheveau de ces interactions multiples, pour progresser dans la compréhension du réacteur atmosphérique et finalement pour améliorer la prévision des phénomènes chimiques atmosphériques, les chercheurs s'appuient sur la synergie de

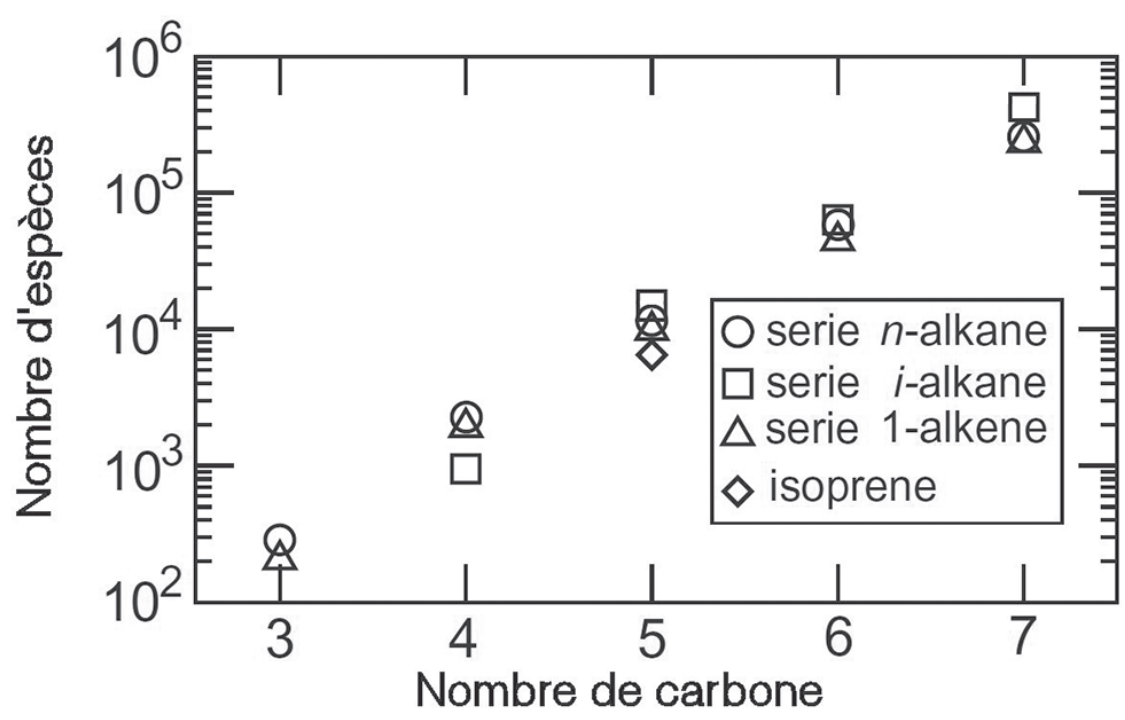

Figure 4. Nombre d'espèces qu'il est nécessaire de prendre en compte pour décrire complètement l'oxydation d'un COV en phase gazeuse selon le nombre de carbones de celui-ci. D'après Aumont et al. (2005). (c) Creative Common License.

trois approches complémentaires : la mesure de terrain, l'étude au laboratoire et la modélisation.

La mesure de terrain et/ou aéroportée - qu'il s'agisse des grandes campagnes communautaires visant à l'exhaustivité des mesures sur un type d'environnement ou d'événement donné ou bien qu'il s'agisse de l'observation de long

terme - constitue souvent le point de départ du questionnement. On y identifie et quantifie les phénomènes que l'on cherche à interpréter, souvent au moyen de modèles de recherche généralement couplant le transport des masses d'air avec les émissions et les transformations chimiques. Des observations inattendues ou à partir de nos difficultés à les reproduire émergent

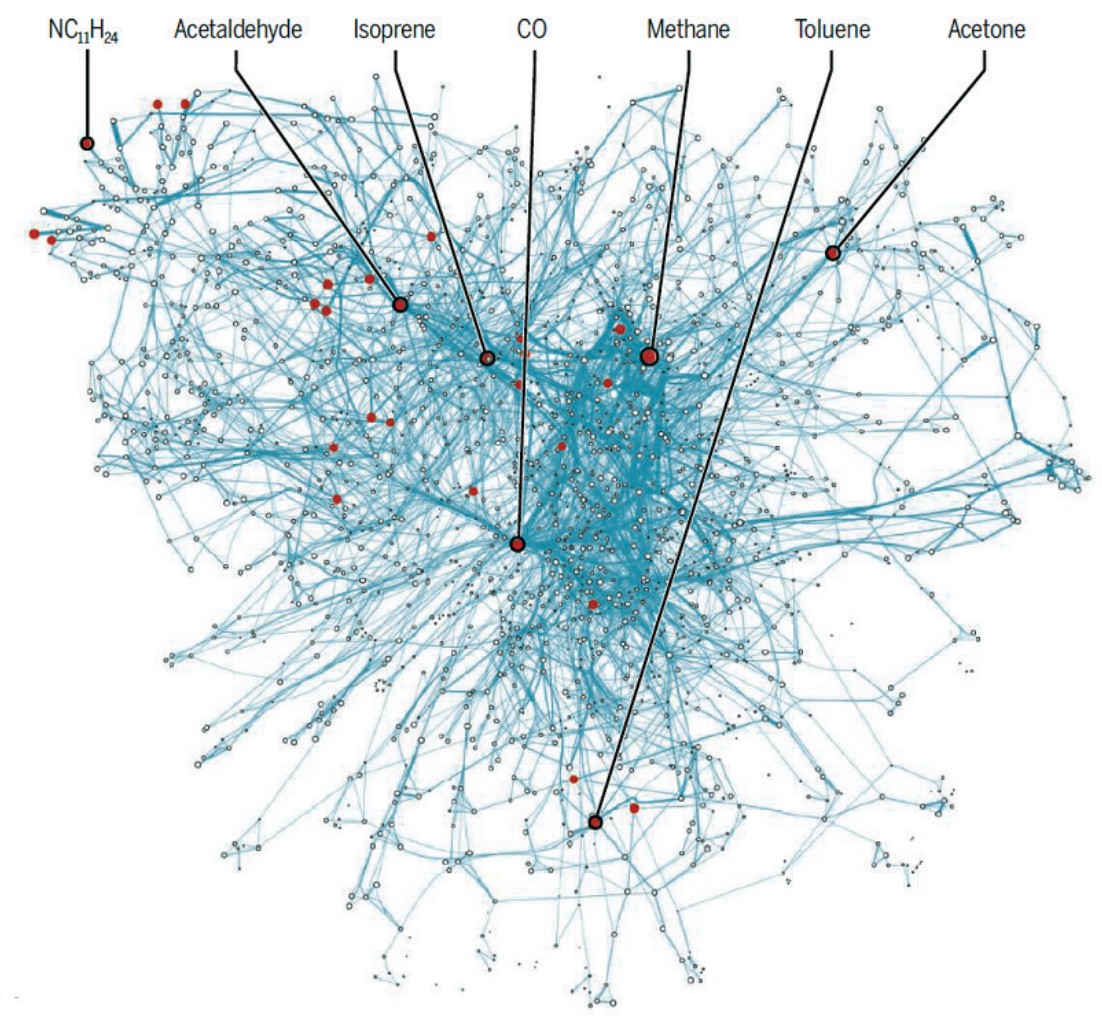

Figure 5. Le réseau complexe des réactions atmosphérique des COV : un petit nombre de composés initialement émis (points rouges) conduit à la formation d'un grand nombre de composés secondaires (points blancs). Les lignes bleues représentent les réactions entre les espèces chimiques telles que prévues par le Master Chemical Mechanism (http://mcm.leeds.ac.uk/MCM/), I'un des modèles de chimie atmosphérique parmi les plus élaborés au monde maintenu par le National Center for Atmospheric Science (NCAS) britannique (d'après Lewis, 2018). (C) AAAS. 
alors des inflexions dans notre questionnement scientifique. Les phénomènes peu compris ou mal quantifiés sont alors étudiés spécifiquement. Le plus souvent, c'est au laboratoire qu'on réussit à les isoler. Chacune des millions de réactions chimiques mentionnées ci-dessus nécessite en effet d'être précisément caractérisée dans ses déterminants et sa vitesse de propagation : c'est le domaine de la cinétique chimique atmosphérique. Pour cela, les chambres de simulation atmosphérique sont des outils de choix. Dans ces réacteurs de grande taille et puissamment équipés des dernières technologies de mesure, on cherche à reproduire de façon maîtrisée les conditions atmosphériques tout en les simplifiant pour comprendre le détail des transformations étudiées. De ces études découlent souvent l'identification de nouveaux processus ou la réévaluation de leur intensité, et ces connaissances nouvelles permettent d'améliorer les modèles qui peuvent alors être, de nouveau, confrontés aux mesures de terrain. Les modèles, quand ils sont suffisamment validés, deviennent alors d'extraordinaires moyens d'exploration, de quantification et de hiérarchisation de la multiplicité des voies réactionnelles décrites ci-dessus.

Ce processus itératif est évidemment décrit schématiquement ici et les voies de la recherche s'appuyant sur l'imagination des chercheurs prennent souvent des tours bien moins balisés. Cependant, cet aller-retour entre les différentes approches, leur interdépendance et leur complémentarité conduit effectivement à la production de connaissances nouvelles quant au fonctionnement chimique de l'atmosphère en tant que compartiment essentiel du système Terre.

Par ailleurs, en améliorant les modèles pour les besoins de la recherche, se crée tout un mouvement qui au final conduit à l'amélioration des modèles opérationnels de qualité de l'air. Enfin, en développant les technologies pour la mesure de terrain ou au laboratoire, la recherche jette les bases de l'instrumentation qui équipe, à terme, les réseaux de surveillance de la qualité de l'air. En identifiant les sources et les processus responsables de la dégradation de la qualité de l'air, elle crée le corpus de connaissance censé éclairer les décideurs. La recherche académique contribue ainsi fortement à répondre à la demande sociétale légitime de respirer un air qui ne nuise pas à la santé.

\section{Bibliographie}

Aumont B., Szopa S., Madronich S., 2005. Modelling the evolution of organic carbon during its gas-phase tropospheric oxidation: development of an explicit model based on a self generating approach. Atmos. Chem. Phys., 5, 2497-2517.

Bates D.V., 2002. Recollections of the London fog. Environ. Health Perspect., 110, A735.

Coll I., Rousseau C., Barletta B., Meinardi S., Blake D.R., 2010. Evaluation of an urban NMHC emission inventory by measurements and impact on CTM results. Atmos. Environ., 44, 3843-3855.

Lewis A., 2018. The changing face of urban air pollution. Science, 359, 6377. doi: 10.1126/science.aar4925 